\title{
Non-Surgical Orthodontic Treatment of Class III Adult with Posterior Crossbite, Anterior Open Bite and Asymmetric Occlusion, Using TADs and Auxiliary Wires: A Case Report
}

\author{
George Philip Dahabreh \\ Department of Orthodontics, Post-Graduate Program, Arab American University, Ramallah, Palestine \\ Email: georgedahabrah1@hotmail.com
}

How to cite this paper: Dahabreh, G.P. (2019) Non-Surgical Orthodontic Treatment of Class III Adult with Posterior Crossbite, Anterior Open Bite and Asymmetric Occlusion, Using TADs and Auxiliary Wires: A Case Report. Open Journal of Stomatology, 9, 227-240.

https://doi.org/10.4236/ojst.2019.910024

Received: September 14, 2019

Accepted: October 13, 2019

Published: October 16, 2019

Copyright $\odot 2019$ by author(s) and Scientific Research Publishing Inc. This work is licensed under the Creative Commons Attribution International License (CC BY 4.0).

http://creativecommons.org/licenses/by/4.0/

\begin{abstract}
Class III malocclusion associated with posterior crossbite, anterior open bite pattern with asymmetric occlusion in adults can be a challenging orthodontic problem, especially for the nonsurgical treatment. Skeletal Class III anteroposterior discrepancies in adult patients are generally managed either by surgical-orthodontic treatment or by orthodontic camouflage through dentoalveolar compensation. Although correction with surgery may be the most effective and stable way, many patients refuse surgical treatment plan because of the costs and traumas it may bring. This case report describes the successful use of TADs with expansion auxiliary wire to treat a 24-year-old man with skeletal class III malocclusion, posterior crossbite, anterior open-bite and asymmetric occlusion, mild upper and lower dental spacing and a chief manifestation of anterior crossbite. The patient refused surgery. A treatment plan was formulated consisting of using auxiliary expansion wire to expand the maxillary arch, $8 \mathrm{~mm}$ mini-screws between the roots of the mandibular canines and first premolars, preadjusted edgewise brackets to align the teeth, Class III and asymmetric elastics to correct the canines, premolars, and molars relationship and midline deviation, reverse curve of the nickel-titanium wire combined with anterior vertical elastics to intrude molars and correct the anterior open-bite. In this case, without going through surgery, the posterior crossbite was corrected, and ideal overjet and overbite relationships, midline coincidence and functional occlusion were all achieved. Satisfactory occlusal, functional, esthetic and stable results were obtained.
\end{abstract}

\section{Keywords}

Non-Surgical, Class III Malocclusion, Orthodontic Treatment, Posterior 


\section{Introduction}

The prevalence of class III malocclusion varies greatly in different racial groups among and within populations (from $1 \%$ to more than $10 \%$ ). The greatest incidence is found among Asian people [1]. Chinese and Malaysian populations show a relatively higher prevalence of angle class III malocclusion $(15.69 \%$ and $16.59 \%$, respectively), while Indian populations show a relatively lower prevalence as compared to other races [2]. In the United States, the prevalence of class III malocclusion is only about $1 \%$ of the total population and only $5 \%$ of orthodontic patients [3].

The number of adult individuals seeking treatment is increasing due to social and esthetic awareness. Skeletal Class III malocclusion is one of the biggest challenges faced by orthodontists [4] [5]. Skeletal Class III patients have anteroposterior, vertical and transverse discrepancies along with dental compensation [6]. Usually it is combined with several other abnormalities such as anterior or posterior crossbites, retroclined mandibular incisors, proclined maxillary incisors, and functional slides from centric relation to centric occlusion. Class III malocclusion associated with skeletal anterior open bite pattern in adults can be a challenging orthodontic problem, especially for non-surgical treatment [7] [8]. Skeletal class III patients can be managed by either orthopedic, orthodontic, camouflage, or combined surgical treatment depending on the age of the patient, the pattern of malocclusion and its severity [9]. In a study on the components of class III surgical patients conducted by Ellis and McNamara [10], it was found that the largest group of the sample, 55\%, was made up of a combination of an underdeveloped maxilla and overdeveloped mandible. If patients consent to orthognathic surgery, subsequent mechanical orthodontic treatment becomes simple with superior functional and esthetic results [11]. However, several patients refuse surgery. In such situations, orthodontic camouflage treatment may be an alternative, particularly if discrepancy is slight or moderate [12]. The introduction of skeletal anchorage has increased the number of patients with skeletal problems who can be treated by mechanical orthodontic treatment only, thereby avoiding the need for complementary orthognathic surgery [13]. Mini-implants are preferred for patients with slight to moderate discrepancies because of less invasive insertion and removal procedures [14] [15].

In the present study, we report the case of a 24 -year-old man with severe skeletal Class III malocclusion who was treated by orthodontic camouflage treatment with mini-implant anchorage and auxiliary wires.

\section{Case Report}

The patient was a 24-year-old male with a concave profile and a normo-divergent 
pattern. (Figure 1). His chief complaint was anterior crossbite and open-bite with spaced dentition. There were no significant findings in his medical and dental histories. The patient had complete dentition without third molars.

Clinical evaluation indicated a dental Class III pattern on both sides, more pronounced on the right side and a midline shift of the mandible by $3.5 \mathrm{~mm}$ to the left. He has no functional shift. Bilateral posterior crossbite, anterior open-bite of $2 \mathrm{~mm}$. He has upper median diastema and lower mild anterior spaces (Figure 2). He denied ever having had any temporomandibular joint dysfunction signs or symptoms, and maximal opening and lateral and anterior movements were within normal limits. Also, there were no deviations on opening and closure, and no joint sounds. The panoramic radiograph showed a complete dentition with missing third molars. The condyles appeared normal in size and form. Dental root lengths and bone heights were normal. Cephalometric analysis confirmed the skeletal Class III pattern; (SNA angle) $77^{\circ}$, (SNB angle) $83^{\circ}$, (ANB angle) $-6^{\circ}$ and Wits of $-5^{\circ}$. Proclined upper incisors, (U1 to SN) $114^{\circ}$. Mildly retroclined lower incisors, (L1-Mand.plane) $88^{\circ}$. Occlusal plane cant, (OP to $\mathrm{SN}) 10^{\circ}$. Normo-divergent facial pattern, (Go-Gn to SN) $29^{\circ}$. Retrusive upper lip, (upper lip to E-plane) $9 \mathrm{~mm}$. (Holdaway angle) $3^{\circ}$ (Figure 3 ).

\subsection{Treatment Objectives}

1) Establish a Class I molar and canine relationship. 2) Correct anterior and

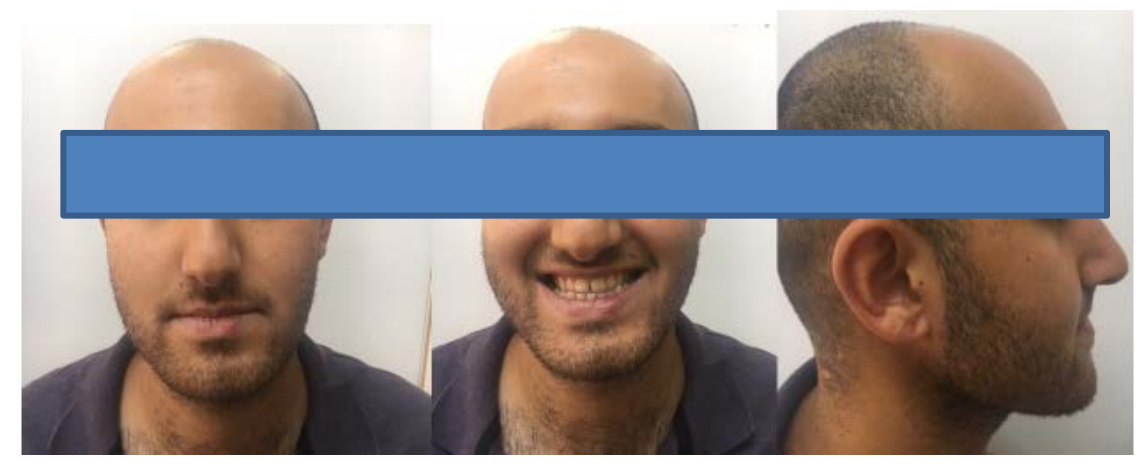

Figure 1. Pretreatment facial photographs, from left to right: the frontal view; the frontal smile view; the profile view.

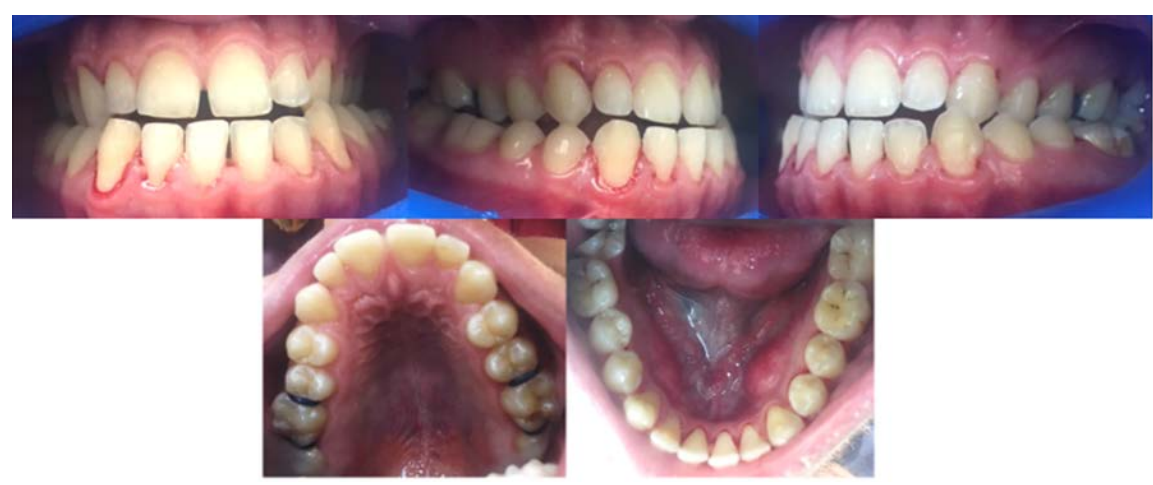

Figure 2. Pretreatment intra-oral photographs. 


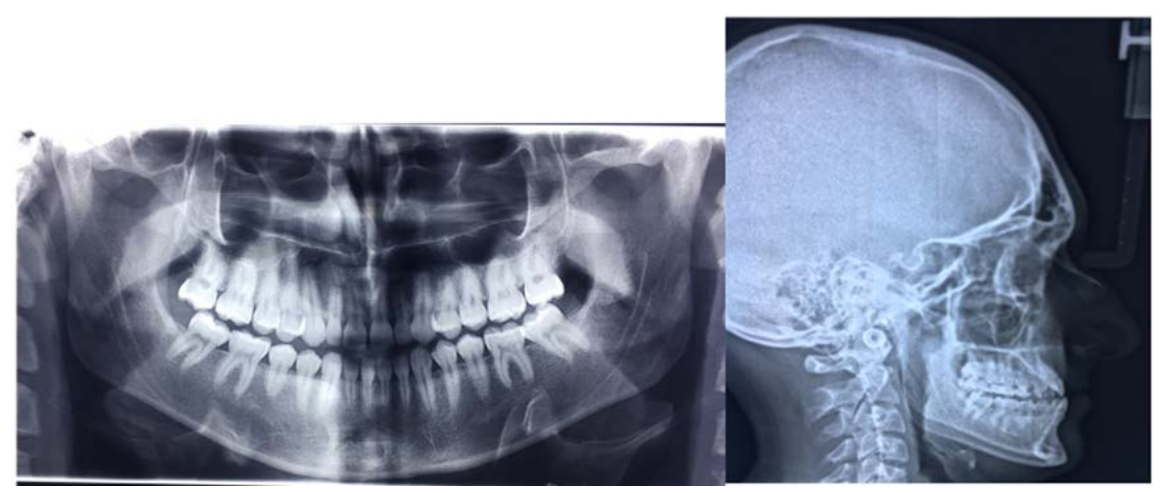

Figure 3. Pretreatment panoramic (left) and cephalometric (right) radiographs.

posterior crossbite to get a harmonious maxillary and mandibular arches width; 3) Achieve adequate overjet and overbite; 4) Correct the midline discrepancy and the asymmetric occlusion. 5) Improve facial esthetics by straightening the facial profile and increasing maxillary incisor exposure at smiling.

\subsection{Treatment Alternatives}

In order to achieve the treatment objectives four possibilities were considered and discussed with the patient.

The first alternative was combined orthognathic surgery and orthodontic treatment, by surgical maxillary advancement with a segmental Le-Fort I, which would certainly improve facial esthetics and simplify subsequent mechanical orthodontic treatment; however, the patient refused to undergo surgery due to costs and risks.

The second alternative was surgically assisted rapid palatal expansion (SARPE), with or without infra-zygomatic TADs, combined with orthodontic treatment and protraction facemask [16]. The patient refused the surgery and the extraoral appliance.

The third alternative was mini-screws assisted rapid palatal expansion (MARPE) combined with orthodontic treatment and miniplates placed in the maxillary zygomatic area and in the anterior area of the mandible and class III mechanics [17]. The patient wanted the least invasive procedure, so, he refused this alternative.

The extraction option for this patient (extracting the mandibular right premolar), and class III mechanics which would have treated the mandibular midline deviation with class III dental relationship was excluded because the lower incisors were retroclined in the first place and using elastics with extraction protocol would cause future dehiscence and fenestrations. Besides, this patient had maxillary deficiency which needed to be addressed.

The fourth alternative was a camouflage treatment. This approach was explained to the patient. After extensive explanation of the use of mini-implants in assisting anchorage in our treatments, he accepted this option. For the first stage, the goal of treatment was to expand maxillary arch to match the mandibular 
arch using expanded auxiliary heavy wire. Thus, posterior crossbite could be corrected. During the second stage, the goal was to create an ideal overbite and overjet relationship and Class I canine and molar relationships, which would be obtained by Class III, cross and asymmetric elastics. To use TADs in the anterior mandibular region to assist in maxillary dental arch protraction and to decrease the adverse effect of expansion on the position of the upper incisors which were spaced, and also to decrease the dependence on the lower dental arch in class III mechanics knowing that the lower incisors are already retroclined. Also, in this stage, reverse curve of the nickel-titanium wire in the upper arch, combined with anterior vertical elastics would be used to help with molars intrusion to create an ideal overbite relationship and to compensate for the extrusive forces used during expansion and protraction. In the third stage, the goal was to maintain the improvement gained in the first and second stages, and to achieve a functional occlusion

\subsection{Treatment Progress}

After obtaining informed consent, treatment began by banding the upper first molars with triple tubes MBT 0.022 first molar Bands. An auxiliary $0.019 \times$ 0.025 -inch stainless steel expanded wire with stopped loops (Piggyback) was inserted in the headgear tubes of the upper first molar band. This auxiliary wire was designed by not applying forces on the upper anterior teeth to prevent retraction forces during expanding the posterior segments. Upper dental arch was not, at this stage, bonded. Two occlusal bite turbos were constructed, with Fuji II type II glass ionomer cement (GC American, Alsip III) on the maxillary second molars to increase the intermaxillary space to allow expansion of the constricted upper dental arch with no intercuspal resistance. This stage lasts for six weeks. After six weeks the mandibular arch was banded and bonded with MBT preadjusted edgewise appliance $(0.022 \times 0.028$ inch $)$ on all teeth including second molars. Initial leveling was accomplished in 3 months with from 0.014 -inch inch round nickel-titanium wires to 0.018 -inch round stainless-steel wires, during which time maxillary dental arch expansion was also accomplished by the stopped piggyback wire assisted by cross elastics (3/16 medium, 3M Unitek, Monrovia, Calif), between the lingual cleats of the upper first molar bands the lower first molar bands hooks (Figure 4). The midline deviation was corrected by applying asymmetrical elastics (1/4 medium.3M Unitek, Monrovia, Calif), between the upper right first molar and lower right canine, keeping the cross elastics on both sides to prevent occlusal plane canting (Figure 5).

After expanding the maxillary molars, MBT pre-adjused edgewise brackets $(0.022 \times 0.028$-inch $)$ were bonded on upper maxillary teeth and upper second molars were banded. Archwire sequence started from 0.014-inch nickel-titanium round wire to $0.019 \times 0.025$-inch stainless steel. In the mandibular arch $0.019 \times$ 0.025 -inch stainless steel was ligated. At this stage two inter-radicular temporary anchorage system $(1.4 \times 8-\mathrm{mm}$; Vector Tas, Ormco, Glendora, Calif) were inserted under local anesthesia between the roots of the lower canines and first 


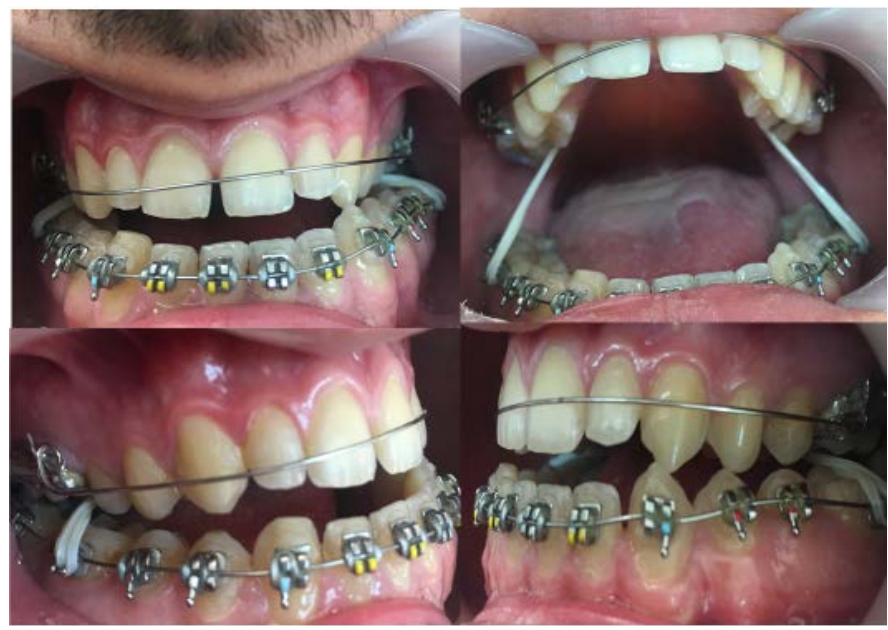

Figure 4. Cross-elastics to assist in expansion of the maxillary arch.

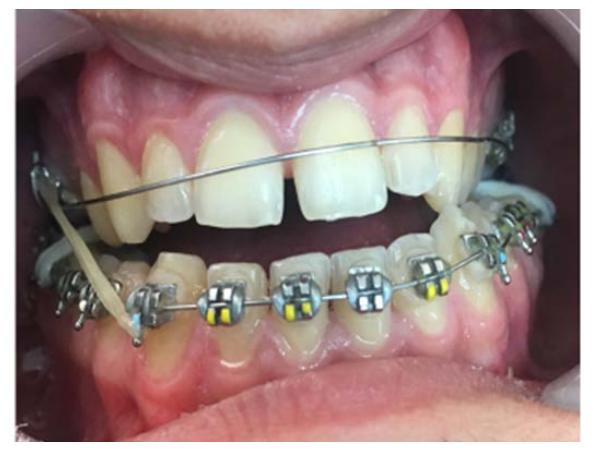

Figure 5. Asymmetric elastics to correct the midline deviation and asymmetric occlusion.

premolars. Protraction of the maxillary dental arch was performed by direct full time engagement of $10 \mathrm{~mm}$ double ended closed nickel-titanium coil spring (Vector, Ormco, Mexico) (225 g-load per side) (Figure 6). This measure was performed for 6 weeks then the patient was instructed to use continuously class III elastics (1/4 Heavy elastics, 6 oz, 3M Unitek, Monrovia, Calif) for another 2 months.

The protraction forces had extrusive forces on the maxillary posterior teeth, and since this patient had an anterior open-bite counteract measures were done to intrude the posterior teeth and correct the anterior openbite. The turbos on the upper second molars were removed. In the upper arch $0.019 \times 0.025$-inch nickel-titanium wire with reverse curve and tip-back bends was used. Vertical elastics (1/4 Heavy elastics, $6 \mathrm{oz}, 3 \mathrm{M}$ Unitek, Monrovia, Calif)) were used by the patient, in a continuous manner, between the upper canines hook and the lower Tads (Figure 7).

To settle the occlusion box elastics were used with class III vector on the right side and class II vector on the left side to adjust the occlusal asymmetry and midline deviation. (1/4 Heavy elastics, 6 oz, 3M Unitek, Monrovia, Calif), were used by the patient all the time except when eating till a well settled and good functional occlusion was accomplished (Figure 8). 


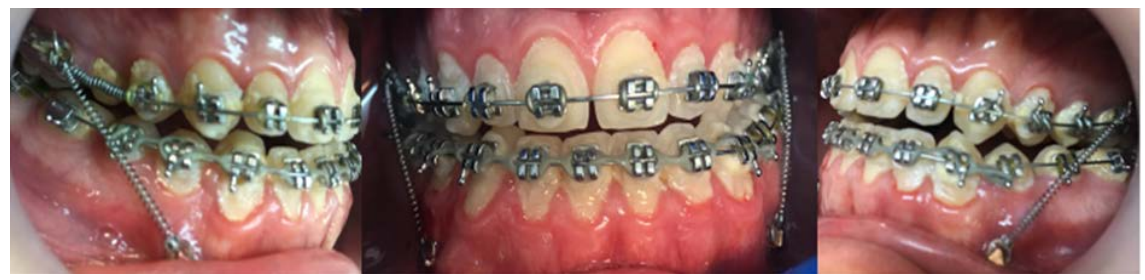

Figure 6. TADs inserted between the roots of the mandibular canines and first premolars, as anchor units used to protract the maxillary dentition applied by full time double ended closed coil spring.

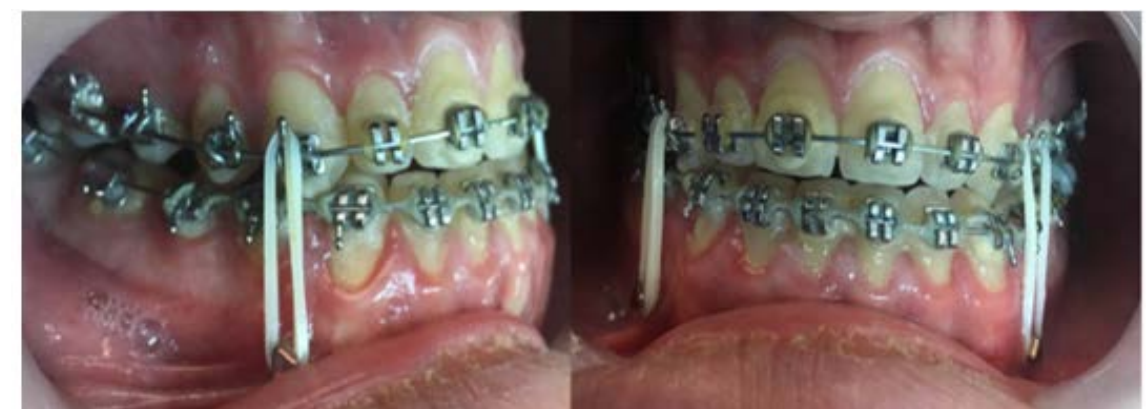

Figure 7. Vertical elastics between the upper canines and the lower TADs to close the anterior openbite.

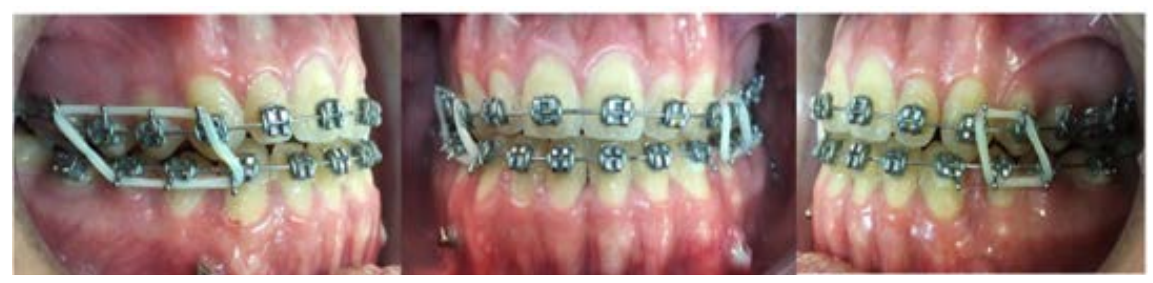

Figure 8. Box elastics with class III vector on the right side and class II vector on the left side.

\subsection{Treatment Results}

After 15 months of active treatment the fixed appliances were removed. Lower and upper 3-3 fixed retainers were bonded. All the treatment objectives were achieved. The anterior crossbite and open-bite were resolved. Posterior crossbite was also corrected. Class I molar and canine relationships were established. Positive overjet, and solid intercuspation was achieved. The dental midlines were coincident with each other and the facial midline (Figure 9). The facial profile and smile were improved and the lower facial height was only slightly increased (Figure 10) Panoramic radiograph showed no root resorptions and well-maintained bone heights. The panoramic radiograph showed also the uprighting of the lower molar teeth. The cephalometric analysis showed improvement in the skeletal class III pattern; (SNA angle) $78^{\circ}$, (SNB angle) $81^{\circ}$, (ANB angle) $-3^{\circ}$, and Wits of -1 $\mathrm{mm}$. Vertical dimensions were slightly increased (Go-Gn to SN $31.5^{\circ}$ ), Slight increase in the lower face height and backward rotation of the mandible. Upper incisors were maintained in their initial proclination angle (UI to SN $112^{\circ}$ ). Retroclination of the lower incisors from start of treatment, (L1-Mand.plane) 


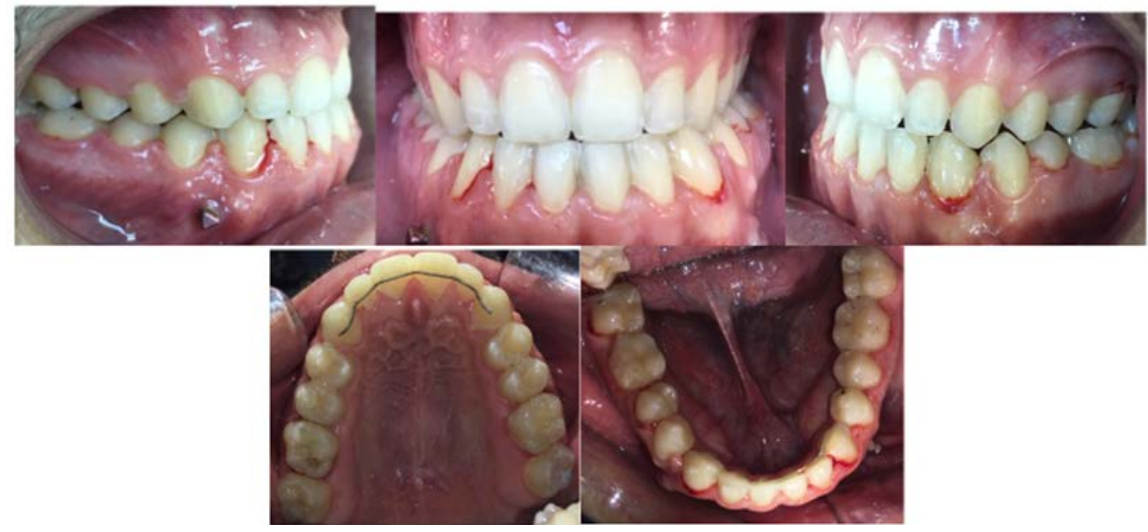

Figure 9. Post-treatment intraoral photographs.

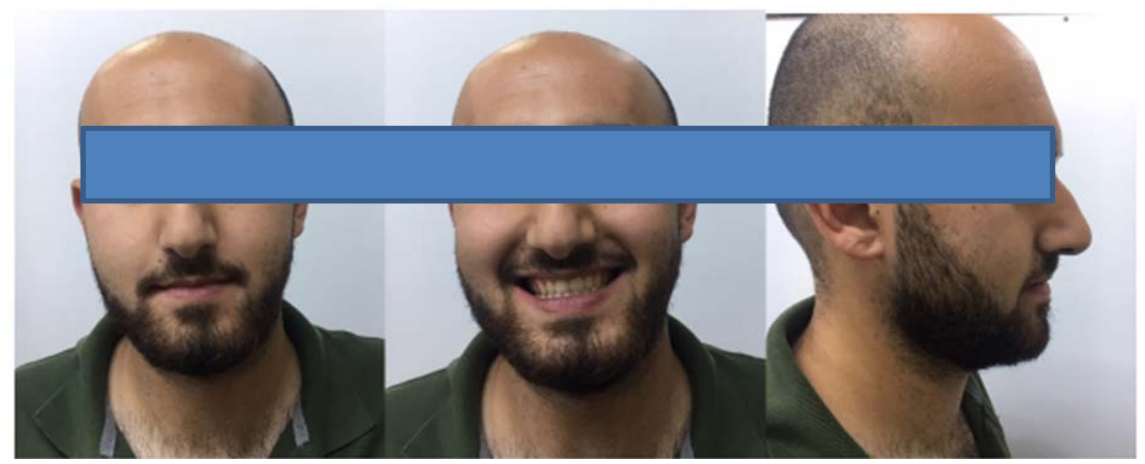

Figure 10. Post-treatment extraoral photographs, from left to right; the frontal view; the frontal smile view; the profile view.

$84^{\circ}$. Inter-incisal angle $137^{\circ}$ (increased slightly). Counter-clockwise rotation of the occlusal plane, (OP to $\mathrm{SN}$ ) $6^{\circ}$. Upper lip to E-plane $-7 \mathrm{~mm}$ (improved), Holdaway angle $5.5^{\circ}$ (improved) (Figure 11). The temporomandibular joints were asymptomatic, and the patient was satisfied with the functional and esthetic results. The posttreatment follow-up of the patient after I year showed stable occlusal and facial results (Figures 12-14). Cephalometric measurements are presented in Table 1.

\section{Discussion}

Studies on the multifactorial etiology of Class III malocclusion show that maxillary retrognathism is as common as mandibular prognathism. Previous research has reported that $32 \%$ - $63 \%$ of the patients with skeletal class III malocclusion as common as mandibular prognathism. Previous research has reported that $32 \%-63 \%$ of the patients with skeletal Class III malocclusion have a maxillary deficiency or its combination with excessive mandibular growth [18].

The severity of class III malocclusion in adult cases would define whether the patient is suitable for surgery or orthodontic treatment [19]. Kerr et al. [20] suggested that surgery should be performed in patients with ANB and incisor mandibular plane angles of lower than $-4^{\circ}$ and $83^{\circ}$, respectively. Rabie et al. [21] 


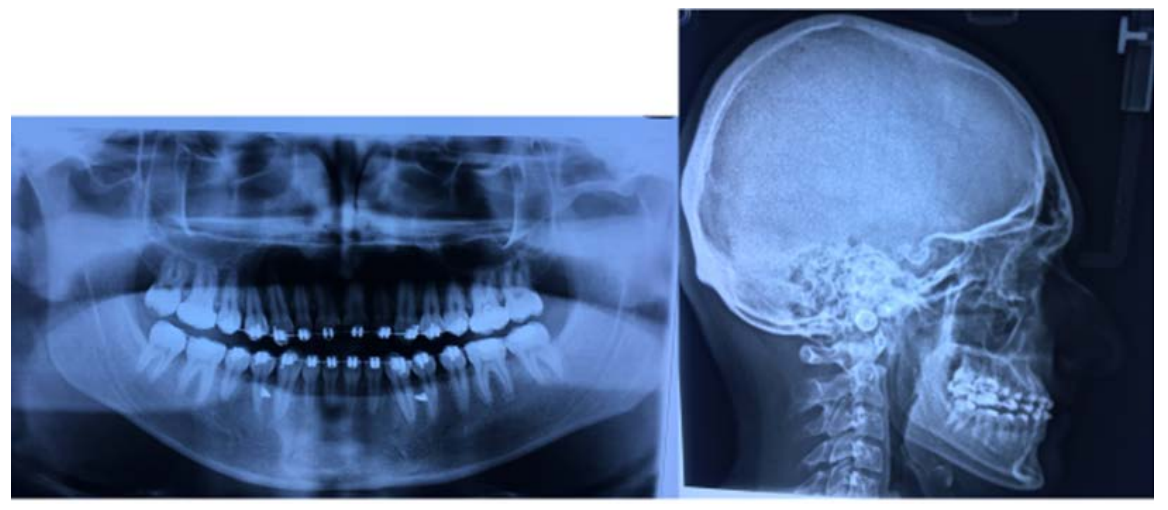

Figure 11. Panoramic (left) and cephalometric (right) radiographs taken one week before debonding.

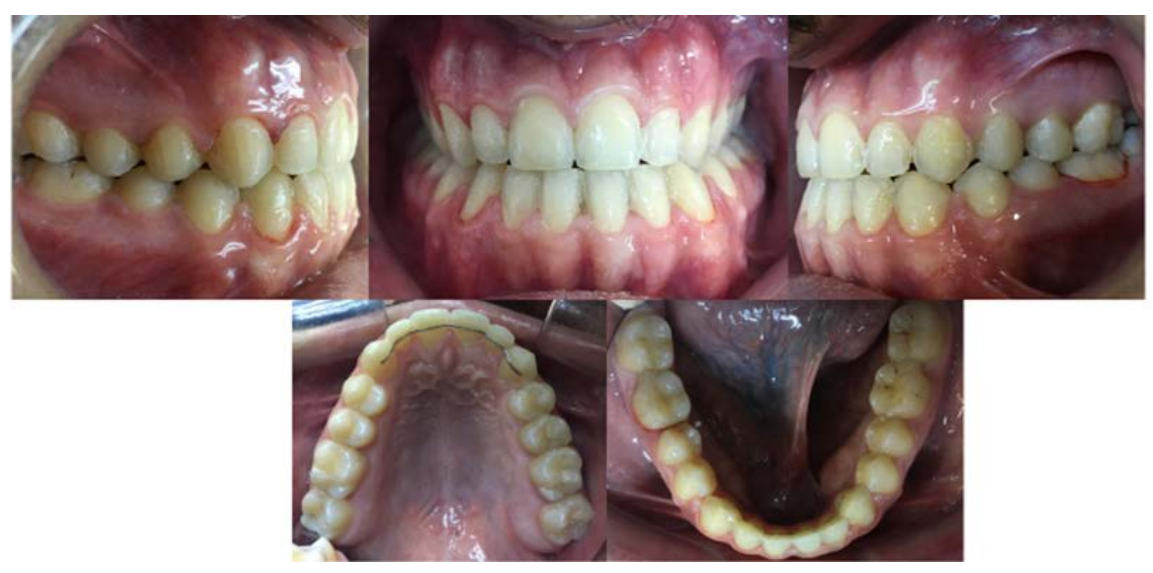

Figure 12. Intraoral photographs one year after treatment.

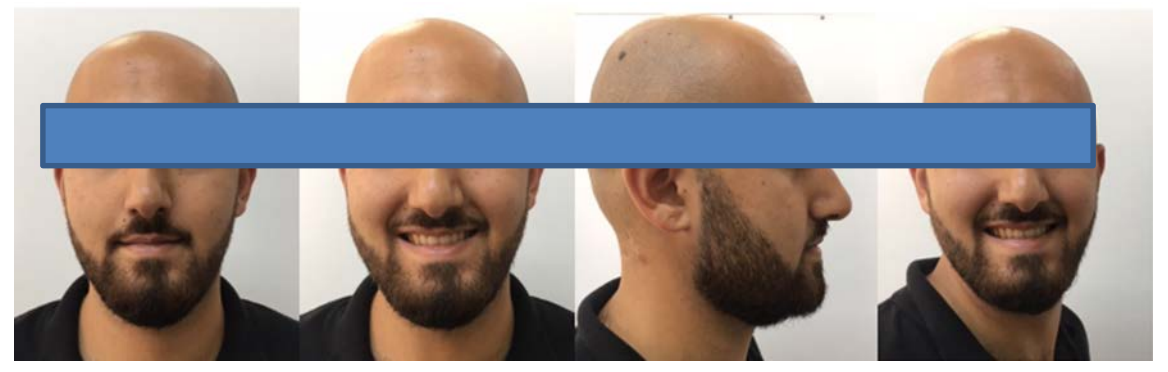

Figure 13. Extraoral photographs one year after treatment.

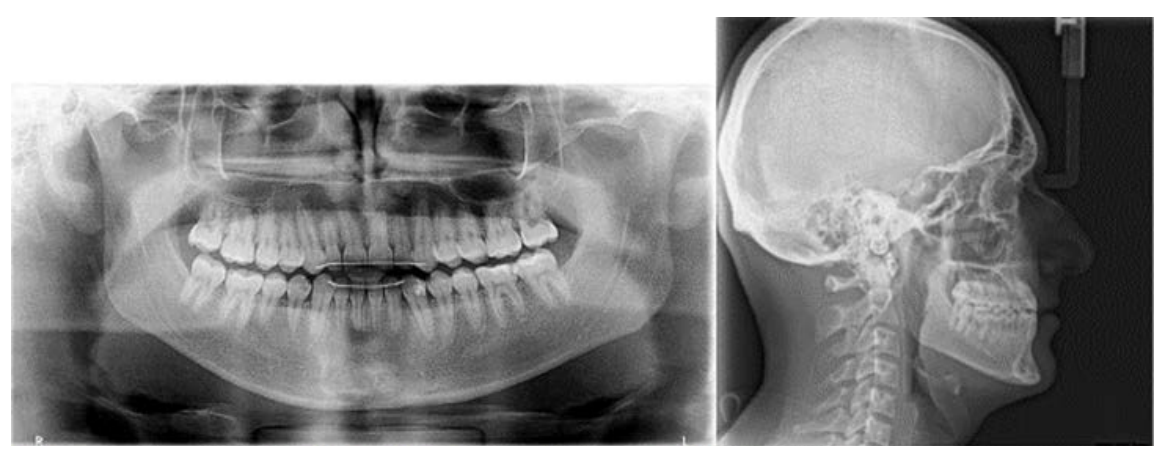

Figure 14. Panoramic (left) and cephalometric (right) radiographs one year after treatment. 
Table 1. Cephalometric measurements.

\begin{tabular}{cccc}
\hline Measurement & initial & After treatment & 1 year retention \\
\hline SNA $\left(^{\circ}\right)$ & 77 & 78 & 77.5 \\
SNB $\left(^{\circ}\right)$ & 83 & 81 & 81 \\
ANB $\left(^{\circ}\right)$ & -6 & -3 & -3.5 \\
Wits $(\mathrm{mm})$ & -5 & -1 & -2 \\
SN-MP $\left({ }^{\circ}\right)$ & 29 & 31.5 & 30.5 \\
U1-SN $\left(^{\circ}\right)$ & 114 & 112 & 111 \\
IMPA $\left(^{\circ}\right)$ & 88 & 84 & 83 \\
OP-SN $\left(^{\circ}\right)$ & 10 & 6 & 7 \\
UL-E plane $(\mathrm{mm})$ & -9 & -7 & -7 \\
LL-E plane $(\mathrm{mm})$ & -5 & -6 & -5.5 \\
Holdaway $\left({ }^{\circ}\right)$ & 3 & 5.5 & 6 \\
\hline
\end{tabular}

evaluated borderline class III patients who had undergone camouflage orthodontic treatment or orthognathic surgery and suggested that Holdaway angle can be a reliable guide in determining the treatment modality of these patients. Lately, Islami et al. [22], concluded that Holdaway angle and Wits appraisal were able to differentiate between the patients suitable for orthodontic camouflage or surgical treatment. Cases with a Holdaway angle greater than $10.3^{\circ}$ and Wits appraisal greater than $-5.8 \mathrm{~mm}$ would be treated successfully by camouflage, while those with a Holdaway angle of less than $10.3^{\circ}$ and with Wits appraisal less than $-5.8 \mathrm{~mm}$ can be treated surgically

For this patient, the etiology of his Class III malocclusion was expressed through maxillary retrusion and mandibular protrusion. He had ANB $-6^{\circ}$, Wits $-5 \mathrm{~mm}$ and Holdaway angle of $3^{\circ}$. According to the previous studies, this patient is a surgical case. The treatment of this case was a three-dimentional planes of treatment which needed the temporary anchorage system to counteract the adverse effects of class III mechanics. This patient, not only had a retrusive maxilla but also a constricted one combined with proclined upper incisors, median diastema and openbite with increased nasolabial angle The lower incisors were retroclined and spaced. Having these data, maxillary expansion, with the presence of the anterior spacing, alone, will retract the maxillary incisors, and thus increase the anterior crossbite. On the other hand using class III mechanics directly to the lower incisors will retrocline them severely, while they are already retroclined and mildly spaced.

The anteroposterior position of the maxillary incisors is important for the harmony of the face and the beauty of the smile. Andrews and Andrews standardized the orthodontic esthetic analysis according to the position of the upper central incisors [23]. Many studies found that the maxillary incisor that is upright or in a slight lingual inclination is preferable [24]. Labioversion of the maxillary incisors can easily ruin a pleasing smile, especially for a patient with a 
Class III facial type [24].

Traditional orthodontic camouflage of skeletal Class III malocclusion always results in more lingual inclination of the mandibular incisors and more proclination of the maxillary incisors for compensation of a skeletal discrepancy [25]. However, excessive inclination of the incisors will not only ruin the dental esthetics but also harm the surrounding periodontal tissues, probably from the traumatic occlusal forces [26]. Unlimited movement of the mandibular incisors is not possible because of the restriction imposed by the symphyseal bone [27]. Because the bone support in the anterior region of a dental arch is limited in skeletal Class III patients, severe iatrogenic sequelae of orthodontic treatment should be considered, consisting of resorption of the labial cortical plate with subsequent gingival recession, dehiscence, or fenestration and lengthening of the clinical crowns of the mandibular incisors or resorption of their lingual cortical plates.

The changes contributing most to the correction of this patient initial dental and skeletal discrepancie were dentoalveolar compensation with en-masse protraction movement of the maxillary dentition and uprighting of the mandibular molars with distal movement of the mandibular dentition: a combination of clockwise rotation of the mandible and counterclockwise rotation of the occlusal plane. The downward and backward movement of the chin expressed in this patient has been described by Ishii et al. [28] and Takada et al. [29]. with maxillary protraction and chincup, and Ngan et al. [30], with maxillary protraction and facemask. The retropositioning of the mandible and counterclockwise rotation of the mandibular plane result in a shortening of the musculature involved in the floor of the mouth, including the genioglossus, geniohyoid, mylohyoid, and digastric muscles. As these muscles become more relaxed, they create a larger space for the base of the tongue in the posterior third of the pharyngeal cavity, which is a naturally comfortable and more functional zone. The anterior pressure of the tip of the tongue against the lower incisors is thereby reduced, resulting in an improvement of the facial profile [31].

Ideal overjet and overbite were achieved with controlled labial movement of the maxillary incisors and retraction of the mandibular anterior teeth. The use of TADs in the anterior mandibular region assisted in protracting the maxillary teeth and enabled the mechanics of expansion and protraction to place the upper incisors in an esthetic position. They were protracted bodily and their final angle was decreased from the initial one. Besides, using the TADs in the anterior position of the mandible as anchor units instead of the lower incisors decreased the lower incisors retroclination adverse effects. They were retroclined by $4^{\circ}$, due to the asymmetric class III elastics used to correct the deviated midline.

\section{Conclusion}

Camouflage treatment of class III adult patient with maxillary deficiency and mandibular prognathism, open-bite and asymmetric occlusion, is a challenge for 


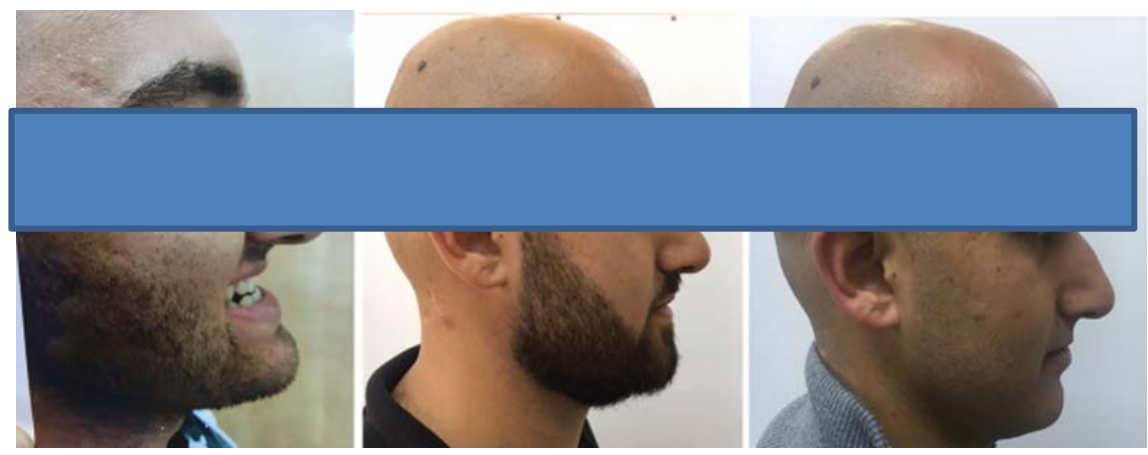

Figure 15. Profile photographs before and one year after treatment.

an orthodontist, but, can be treated successfully, given excellent patient cooperation. In this case report, excellent occlusal and esthetic results were achieved with the aid of auxiliary expansion, reverse curved wires and TADs placed between the roots of the mandibular canines and premolars (Figure 15).

\section{Acknowledgements}

Thanks Dr. Mauricio González Balut for his assistance in digitizing the Cephalometric tracings. I thank the patient for allowing the publication of this case report. Informed consent was obtained from the patient for publication of this case report and any accompanying images.

\section{Conflicts of Interest}

The author declares no conflicts of interest regarding the publication of this paper.

\section{References}

[1] Ishii, I., Morita, S., Takeuchi, Y. and Nakamura, S. (1987) Treatment Effect of Combined Maxillary Protraction and Chincap Appliance in Severe Skeletal Class III Cases. American Journal of Orthodontics and Dentofacial Orthopedics, 92, 304-312. https://doi.org/10.1016/0889-5406(87)90331-3

[2] Soh, J., Sandham, A. and Chan, Y.H. (2005) Occlusal Status in Asian Male Adults: Prevalence and Ethnic Variation. The Angle Orthodontist, 75, 814-820.

[3] Graber, T.M., Vanarsdall, R.L. and Vig, K.W.L. (2005) Orthodontics: Current Principles and Techniques. 4rth Edition, Mosby, St Louis, 565.

[4] Antoszewska, J., Kosior, M. and Antoszewska, N. (2011) Treatment Approaches in Class III Malocclusion with Emphasis on Maximum Skeletal Anchorage: Review of Literature. Journal of Stomatology, 64, 667-683.

[5] Farret, M.M. and Benitez Farret, M.M. (2013) Skeletal Class III Malocclusion Treated Using a Nonsurgical Approach Supplemented with Mini-Implants: A Case Report. Journal of Orthodontics, 40, 256-263. https://doi.org/10.1179/1465313313Y.0000000046

[6] Baik, H.S. (2007) Limitations in Orthopedic and Camouflage Treatment for Class III Malocclusion. Seminars in Orthodontics, 13, 158-174.

https://doi.org/10.1053/j.sodo.2007.05.004 
[7] Nielsen, I.L. (1991) Vertical Malocclusions: Etiology, Development, Diagnosis and Some Aspects of Treatment. The Angle Orthodontist, 61, 247-260.

[8] Lopez-Gavito, G., Wallen, T.R., Little, R.M. and Joondeph, D.R. (1985) Anterior Open-Bite Malocclusion: A Longitudinal 10-Year Postretention Evaluation of Treated Patients. American Journal of Orthodontics, 87, 175-186. https://doi.org/10.1016/0002-9416(85)90038-7

[9] Proffit, W.R., Fields, H.W. and Sarver, D.M. (2007) Orthodontic Treatment Planning; Limitations, Controversies, and Special Problems. In: Proffit, W.R., Fields, H.W., Sarver, D.M., Eds., Contemporary Orthodontics, 4th Edition, Mosby, St Louis, 268-327.

[10] Ellis, E.F. and McNamara, J.A. (1984) Components of Adult Class III Malocclusion. Journal of Oral and Maxillofacial Surgery, 42, 295-305.

https://doi.org/10.1016/0278-2391(84)90109-5

[11] Lin, J. and Gu, Y. (2003) Preliminary Investigation of Nonsurgical Treatment of Severe Skeletal Class III Malocclusion in the Permanent Dentition. The Angle Orthodontist, 73, 401-410.

[12] Kuroda, Y., Kuroda, S., Alexander, R.G. and Tanaka, E. (2010) Adult Class III Treatment Using a J-Hook Headgear to the Mandibular Arch. The Angle Orthodontist, 80, 336-343. https://doi.org/10.2319/041609-218.1

[13] Freire-Maia, B., Pereira, T.J. and Ribeiro, M.P. (2011) Distalization of Impacted Mandibular Second Molar Using Miniplates for Skeletal Anchorage: Case Report. Dental Press Journal of Orthodontics, 16, 132-136. https://doi.org/10.1590/S2176-94512011000400020

[14] Kuroda, S. and Tanaka, E. (2011) Application of Temporary Anchorage Devices for the Treatment of Adult Class III Malocclusions. Seminars in Orthodontics, 17, 91-97. https://doi.org/10.1053/j.sodo.2010.12.002

[15] Sugawara, Y., Kuroda, S., Tamamura, N. and Takano-Yamamoto, T. (2008) Adult Patient with Mandibular Protrusion and Unstable Occlusion Treated with Titanium Screw Anchorage. American Journal of Orthodontics and Dentofacial Orthopedics, 133, 102-111. https://doi.org/10.1016/j.ajodo.2006.06.020

[16] Jackson, G.W. and Kravitz, N.D. (2014) Expansion/Facemask Treatment of an Adult Class III Malocclusion. Case Reports in Dentistry, 2014, Article ID: 270257. https://doi.org/10.1155/2014/270257

[17] Moon, W., Wu, K.W., MacGinnis, M., Sung, J., Chu, H., Yousef, G. and Machodo, A. (2015) The Efficacy of Maxillary Protraction Protocols with the Micro-Implant-Assisted Rapid Palatal Expander (MARPE) and the Novel N2 Mini-Implant: A Finite Element Study. Progress in Orthodontics, 16, 16. https://doi.org/10.1186/s40510-015-0083-Z

[18] Ngan, P. and Moon, W. (2015) Evolution of Class III Treatment in Orthodontics. American Journal of Orthodontics and Dentofacial Orthopedics, 148, 22-36. https://doi.org/10.1016/j.ajodo.2015.04.012

[19] Perillo, L., Monsurro, A., Bonci, E., Torella, A., Mutarelli, M. and Nigro, V. (2015) Genetic Association of ARHGAP21 Gene Variant with Mandibular Prognathism. Journal of Dental Research, 94, 569-576. https://doi.org/10.1177/0022034515572190

[20] Kerr, W.J., Miller, S. and Dawber, J.E. (1992) Class III Malocclusion: Surgery or Orthodontics? British Journal of Orthodontics, 19, 21-24. https://doi.org/10.1179/bjo.19.1.21

[21] Rabie, A.B., Wong, R.W. and Min, G.U. (2008) Treatment in Borderline Class III Malocclusion: Orthodontic Camouflage (Extraction) versus Orthognathic Surgery. 
The Open Dentistry Journal, 2, 38-48. https://doi.org/10.2174/1874210600802010038

[22] Islami, S., Faber, J., Fateh, A., Sheikholaemmeh, F., Grassia, V. and Jamilian, A. (2018) Treatment Decision in Adult Patients with Class III Malocclusion: Surgery versus Orthodontics. Progress in Orthodontics, 19, 28. https://doi.org/10.1186/s40510-018-0218-0

[23] Andrews, L.F. and Andrews, W.A. (2001) Syllabus of the Andrews Orthodontic Philosophy. 9th Edition, Lawrence F. Andrews, San Diego.

[24] Cao, L., Zhang, K., Bai, D., Jing, Y., Tian, Y. and Guo, Y. (2011) Effect of Maxillary Incisor Labiolingual Inclination and Anteroposterior Position on Smiling Profile Esthetics. The Angle Orthodontist, 81, 121-129.

https://doi.org/10.2319/033110-181.1

[25] Bishara, S.E. (2001) Textbook of Orthodontics. $7^{\text {th }}$ Edition, Saunders, Philadelphia, $\mathrm{Pa}, 375-414$.

[26] Handelman, C.S. (1996) The Anterior Alveolus: Its Importance in Limiting Orthodontic Treatment and Its Influence on the Occurrence of Iatrogenic Sequelae. The Angle Orthodontist, 66, 95-109.

[27] Sarikaya, S., Haydar, B., Ciger, S. and Ariyurek, M. (2002) Changes in Alveolar Bone Thickness Due to Retraction of Anterior Teeth. American Journal of Orthodontics and Dentofacial Orthopedics, 122, 15-26. https://doi.org/10.1067/mod.2002.119804

[28] Ishii, H., Morita, S., Takeuchi, Y. and Nakamura, S. (1987) Treatment Effect of Combined Maxillary Protraction and Chin-Cap Appliance in Severe Skeletal Class III Cases. American Journal of Orthodontics and Dentofacial Orthopedics, 92, 304-312. https://doi.org/10.1016/0889-5406(87)90331-3

[29] Takada, K., Petdachai, S. and Sakuda, M. (1993) Changes in Dentofacial Morphology in Skeletal Class III Children Treated by a Modified Maxillary Protraction Headgear and a Chin Cup: A Longitudinal Cephalometric Appraisal. European Journal of Orthodontics, 15, 211-221. https://doi.org/10.1093/ejo/15.3.211

[30] Ngan, P., Urban, H., Yiu, C., Merwin, D. and Wei, S. (1996) Soft Tissue and Dentoskeletal Profile Changes Associated with Maxillary Expansion and Protraction Headgear Treatment. American Journal of Orthodontics \& Dentofacial Orthopedics, 109, 38-49. https://doi.org/10.1016/S0889-5406(96)70161-0

[31] Carriere, L. (2016) Nonsurgical Correction of Severe Skeletal Class III Malocclusion. Journal of Clinical Orthodontics, 50, 216-230. 